\title{
nano dossiers
}

Institut für Technikfolgen-Abschätzung der

S. Greßler, A. Gazsó*, M. Simkó, M. Nentwich, U. Fiedeler

\section{Nanopartikel und nanostrukturierte Materialien in der Lebensmittelindustrie}

\section{Zusammenfassung}

Nanotechnologie bietet für die Lebensmittelindustrie interessante Anwendungsmöglichkeiten und ein großes Marktpotenzial. Dem gegenüber steht aber eine vorwiegend ablehnende Einstellung der KonsumentInnen zu Nanomaterialien und Nanopartikel in Lebensmitteln. Hersteller kommunizieren zu diesem äuBerst sensiblen Thema deshalb nur wenig. Daraus resultiert ein derzeit sehr geringer Informationsstand zu Entwicklungen und tatsächlichen Anwendungen. Synthetische Nanomaterialien in Form von Nanokapseln oder Nanoemulsionen finden als Trägersysteme und zum Schutz aktiver Inhaltsstoffe bei der Herstellung, Lagerung und im Handel besonderes Interesse. Nanopartikuläre Farbstoffe werden bei der Getränkeherstellung eingesetzt, nanoskalige Kieselsäure findet als Lebensmittelzusatz- und -verarbeitungshilfsstoff Verwendung, Vitamine, Mineralien und Heilkräuter werden in Nanogröße Nahrungsergänzungsmitteln zugefügt. Darüber hinaus findet Nanotechnologie auch Anwendung im Bereich der Lebensmittelverpackung, der Lebensmittelsicherheit und Sensortechnik.

\section{Einleitung}

Das Thema Nanotechnologie im Lebensmittelbereich gewinnt zunehmend an öffentlichem Interesse. So etwa widmet sich der BUND (Friends of the Earth) in seiner kürzlich erschienenen Veröffentlichung "Aus dem Labor auf den Teller" ausführlich der Nutzung von Nanotechnologie im Lebensmittelsektor. Doch fundierte Informationen sind spärlich und viele Fragen insbesondere zum Konsumentenschutz sind noch offen.

Die Nanotechnologie bietet viele neue Möglichkeiten, die auch für die Lebensmittelindustrie von Interesse sind. Die Helmut Kaiser Consultancy prognostiziert in ihrer Studie "Nanofood" (2004) ein Marktpotenzial von 20,4 Mrd. USD für das Jahr 2010. Mehr als 200 Firmen weltweit sollen in diesem Bereich bereits tätig sein, v. a. in den USA, Japan und China. ' So hat z. B. Kraft Foods Inc. (USA) im Jahr 2000 das "Nanotek"-Konsortium ins Leben gerufen, in dem 15 Universitäten und Forschungseinrichtungen weltweit involviert sind. ${ }^{1}$ Mars Inc. (USA) hält ein Patent ${ }^{2}$ für ultradünne anorganische Überzüge von Produkten für den Verzehr. ${ }^{3}$ Nestlé $(\mathrm{CH})$ beschäftigt sich mit Verkapselungssystemen 4 für die Abgabe von aktiven Inhaltsstoffen (z. B. Geschmacksstoffe, Vitamine, Fettsäuren) in Lebensmitteln. ${ }^{5}$

Nanotechnologie im Lebensmittelbereich ist ein sensibles Thema. Hersteller befürchten ähnlich wie im Fall der Gentechnik eine pauschale Ablehnung von Produkten, die Nanomaterialien enthalten. Die Befürchtungen sind nicht unberechtigt, denn jüngste Umfragen zeigen, dass die Mehrheit der Konsumenten und Konsumentinnen Nanopartikel oder Nanomaterialien in Lebensmitteln ablehnen. ${ }^{6}$ Lebensmittelkonzerne kommunizieren deshalb nur in geringem Maße die Verwendung von Nanomaterialien in Produkten. Aus diesem Grund ist nicht bekannt, wie viele solcher Lebensmittel derzeit bereits auf dem Markt sind. Darüber hinaus bestehen noch Un- klarheiten darüber, welche Entwicklungen tatsächlich der Nanotechnologie zuzurechnen sind. Klare Abgrenzungen und Definitionen fehlen - sie festzulegen ist aber aufgrund der Komplexität und Vielgestaltigkeit der Nanotechnologie auch kein leichtes Unterfangen.

Dieses Dossier gibt zunächst einen Überblick über die Grundlagen für die Anwendung von Nanotechnologie im Lebensmittelbereich. Danach werden die verschiedenen Anwendungsbereiche wie Lebensmittelherstellung und Verpackung, Sicherheit und Sensortechnik kurz dargestellt. In einem abschließenden Abschnitt werden Beispiele von Anwendungen und Produkten genannt, die bereits im Handel erhältlich sind.

\section{Grundlagen}

In Lebensmitteln finden sich von Natur aus Bestandteile in Nanometergröße. Diese sind von synthetisch hergestellten Nanomaterialien zu unterscheiden. Weiters werden unsere Lebensmittel schon seit langem Verund Bearbeitungsprozessen unterzogen, bei denen Nanostrukturen entstehen, die aber nichts mit moderner Nanotechnologie zu tun haben, wie etwa Gelieren, Emulgieren oder Homogenisieren. Die beiden folgenden Abschnitte bieten einen kurzen Überblick über die wichtigsten Grundlagen und Definitionen.

\section{Natürliche Nanostrukturen in Lebensmitteln}

Lebensmittelproteine sind globuläre Partikel in Größen von 10 bis einigen hundert Nanometern. Viele Polysaccharide und Lipide sind kettenförmige Polymere, die in einer Dimension weniger als einen Nanometer groß sind. Gelieren oder Emulgieren basieren auf der Bildung von netzartigen, zwei- und dreidimensionalen Nanostrukturen. Wenn Stärke gekocht wird, um Pud- 
ding herzustellen, werden nur einige zehn Nanometer große, dreidimensionale Kristallstrukturen geschmolzen. ${ }^{1}$

Milch und Milchprodukte beinhalten natürlicherweise Nanostrukturen, wie Molkeproteine und Caseine. Bei der Homogenisierung von Milch entstehen Fettkügelchen in der Größe von etwa 100 nm. ${ }^{7}$

\section{Synthetische Nanomaterialien in Lebensmitteln ${ }^{8}$}

Nanotechnologie hat v. a. ein großes Potenzial im Bereich der funktionellen Lebensmittel („Functional Food"9, ,Nutraceuticals"10). Wirkstoffe und Substanzen können in nanostrukturierte Materialien eingeschlossen werden (Verkapselung). Dadurch soll(en)

- die Löslichkeit verbessert werden (z. B. von Farbstoffen),

- eine kontrollierte Abgabe ermöglicht werden (z. B. erst in bestimmten Teilen des Verdauungstraktes, etwa um den schlechten Geschmack eines an sich wertvollen Inhaltsstoffes zu verhindern. Beispiel: Omega-3-Fettsäuren in Fischölen),

- die Bioverfügbarkeit, d. h. die vom Körper tatsächlich aufgenommene Menge eines Nahrungsbestandteils (z. B. Vitamine, Mineralien) erhöht werden,

- Mikronährstoffe und bioaktive Komponenten während der Herstellung, Lagerung und im Handel geschütz† werden.

Die derzeit wichtigsten nanostrukturierten Materialien sind Nanokapseln (Mizellen, Liposome) und Nanoemulsionen.

\section{Mizellen}

Mizellen sind kugelförmige Strukturen mit einem Durchmesser von 5-100 nm. Sie bilden sich spontan, wenn ein Netzmittel (Tensid) in Wasser gelöst wird. Mizellen haben die Fähigkeit, nicht-polare Moleküle, wie etwa Lipide, Geschmackstoffe, antimikrobielle Substanzen, Antioxidantien und Vitamine einschließen zu können. Komponenten, die normalerweise nicht wasserlöslich sind, können mithilfe von Mizellen wasserlöslich gemacht werden. Mizellen, die gelöste Materialien beinhalten, werden als Mikroemulsionen bezeichnet. Mizellen werden in der Pharmazie schon seit längerem verwendet, finden aber erst seit kurzem das Interesse der Lebensmittelindustrie.

\section{Liposome (Lipidvesikel)}

Liposome sind kugelförmige, polymolekulare Aggregate mit einer doppellagigen Schale. Die Größe variiert von 20 nm bis einige hundert Mikrometer.

Liposome werden durch polare Lipide gebildet, die häufig in der Natur zu finden sind (z. B. Phospholipide aus Soja und Eiern). Wie auch Mizellen können Liposome ein breites Spektrum von funktionalen Komponenten umschließen. Der Unterschied ist allerdings, dass Liposome sowohl wasser- als auch fettlösliche Komponenten einkapseln können. Liposome werden erfolgreich eingesetzt, um empfindliche Proteine einzuschließen, wobei diese unabhängig von den äußeren Bedingungen (z. B. chemische Einflüsse) ihre Funktion behalten. Mithilfe von Liposomen kann z. B. auch die Lagerfähigkeit von Milchprodukten erhöht werden.

\section{Nanoemulsionen}

Nanoemulsionen sind sehr feine Öl-in-Wasser-Emulsionen mit einer mittleren Tropfengröße von 50-200 nm. Nanoemulsionen streven das sichtbare Licht nicht, sodass sie transparent sind. Aufgrund der geringen Teilchengröße bleiben Nanoemulsionen auch längere Zeit stabil, d. h. es kommt später als bei herkömmlichen Emulsionen zu einer Trennung von Öl und Wasser (was zu einem kompletten Zusammenbruch der Emulsion führt). Die Bioverfügbarkeit von lipophilen Substanzen kann durch Nanoemulsionen stark erhöht werden. So werden Nanoemulsionen z. B. seit längerer Zeit für die parenterale (künstliche) Ernährung eingesetzt. Auch zeigen sie interessante Textureigenschaften - sie verhalten sich sogar bei einer geringen Öltropfenkonzentration wie eine zähflüssige Creme, was sie interessant für die Entwicklung von fettreduzierten Produkten macht.

\section{Anwendungsbereiche}

Im Folgenden wird eine Auswahl möglicher Anwendungsbereiche von Nanopartikeln und nanostrukturierten Materialien in der Lebensmittelindustrie präsentiert. Neben den Hilfsund Zusatzstoffen zählen dazu auch prozesstechnische Anwendungen. ${ }^{11}$

\section{Lebensmittelherstellung und Zusatzstoffe}

(1) Funktionelle Lebensmittel („,Functional Food" bzw. "Nutraceuticals"): Mizellen und Liposome (Lipidvesikel) können als Träger für ätherische Öle, Geschmackstoffe, Antioxidantien, Coenzym Q10, Vitamine, Mineralien, Pflanzenwirkstoffe, Proteine, Enzyme, Geschmackstoffe und antimikrobielle Komponenten fungieren (Produkt z. B. Canola Active Oil, Israel; Mizellen mit Phytosterol). Durch die Verkapselung aktiver Wirkstoffe (z. B. Polyphenol, Mineralien, Mikronährstoffe) können diese auch vor Oxidation geschützt und ihr schlechter Geschmack im Endprodukt verringert werden (Produkt Tip-Top Up Bread, Australien; Brot mit Nanokapseln für Fischöl, das die gesundheitsfördernden Omega-3-Fettsäuren enthält). Nanoröhren (Nanotubes) und Nanokugeln (Nanospheres) aus Milch- bzw. Molkeprotein können als Geliermittel und ebenfalls für die Verkapselung von Wirkstoffen oder als Trägermaterial zur kontrollierten Abgabe von Substanzen eingesetzt werden (in Entwicklung).

(2) Synthetisches nanopartikuläres Lycopin (ein Carotinoid; roter Farbstoff der Tomaten) als Nahrungsergänzungsmittel und zur Anreicherung in Lebensmitteln (BASF, Produkt LycoVit). Durch die amerikanische Lebensmittelbehörde (US Food and Drug Administration, FDA) als sicher eingestuft („generally recognized as safe", GRAS). Ein Antrag auf Zulassung innerhalb der EU wurde gestellt. ${ }^{12}$

(3) Nanopartikuläres Beta-Carotin als Farbstoff bei der Getränkeherstellung (Produkt Lucarotin 10 CWD, BASF). Ein Schutzkolloid aus modifizierter Stärke umgibt die Nanopartikel und verhindert die Aggregation und Agglomeration („Klumpenbildung“). Carotin und modifizierte Stärke sind zugelassene Lebensmittelzusatzstoffe (E160a bzw. E1450). Dadurch ist die Zulassung des nanopartikulären Carotins gegeben. ${ }^{13}$

(4) Nanopartikuläres Siliciumdioxid $\left(\mathrm{SiO}_{2}\right)$ : Siliciumdioxid ist nur für bestimmte Lebensmittel zugelassen (E551), u. a. für Trockenlebensmittel in Pulverform, Käse (in Scheiben oder gerieben), Würzmittel, Nahrungsergänzungsmittel, Kochsalz, Kochsalzersatz und als Zusatzstoff deklarationspflichtig. AuBerdem ist es als Trägerstoff von Emulgatoren, Farbstoffen und Aromen erlaubt. Die tolerierbare tägliche Aufnahme ist nicht festgelegt; Siliciumdioxid gilt als unbedenklich. ${ }^{14}$ Darüber hinaus darf $\mathrm{SiO}_{2}$ als $\mathrm{Gel}$ oder kolloidale Lösung (fein verteilte Teilchen) auch als Verarbeitungshilfsstoff für Lebensmittel pflanzlichen Ursprungs verwendet werden. ${ }^{15}$ 
Verarbeitungshilfsstoffe sind keine Zutaten, sondern werden bei der technischen $\mathrm{Be}$ - und Verarbeitung verwendet, wobei Rückstände im Endprodukt verbleiben können.

Synthetisches $\mathrm{SiO}_{2}$ wird u. a. als pyrogene (kolloidale) Kieselsäure von verschiedenen Firmen angeboten, z. B. unter dem Produktnamen Aerosil von der Firma Evonik ${ }^{16}$ (Anwendungen: Lebensmittel und Kosmetik; Verdickungsmittel, Verbesserung der Rieselfähigkeit von Pulvern, gegen das Verklumpen von Pulvern). "Bad Ischler 7-Kräuter-Salz” enthält z. B. Aerosil als Rieselhilfe. ${ }^{17}$

Die Primärteilchen der pyrogenen Kieselsäure liegen naturgemäß in Nanogröße vor, da es sich dabei um ein Kolloid handelt. Die Teilchen neigen aber zu Agglomerationen, die größer als $100 \mathrm{~nm}$ sind. Einzelne Partikel treten nur gelegentlich auf, bilden aber Aggregate, die als unbedenklich für die Gesundheit gelten, da sie unverändert ausgeschieden werden. ${ }^{18}$

Synthetische Kieselsäure ist kein Produkt der modernen Nanotechnologie. Sie wird seit vielen Jahren produziert und im Lebensmittelbereich verwendet. Struktur und Teilchenfeinheit wurden nicht verändert. ${ }^{19}$

\section{(5) Nanopartikuläres Titandioxid $\left(\mathrm{TiO}_{\mathbf{2}}\right)$ :} $\mathrm{TiO}_{2}$ ist in der größeren Form ein zugelassener Lebensmittelfarbstoff (E 171). Es wird aufgrund seiner UV-Beständigkeit als weißes Pigment in Papier und Plastik verwendet. $\mathrm{TiO}_{2}$ ist auch direkt in Lebensmitteln zu finden, etwa in der Zuckerglasur von Konfekt oder auch in Instant-Getränken. Mars Inc. (USA) hält ein Patent 20 für anorganische Überzüge von Produkten für den Verzehr. ${ }^{21}$ Insbesondere Süßwaren sollen durch eine transparente, ultradünne Schicht $(0,2-500 \mathrm{~nm})$ von anorganischem Material $\left(\mathrm{SiO}_{2}, \mathrm{SiO}\right.$, $\mathrm{CaO}, \mathrm{ZnO}, \mathrm{TiO}_{2}$ oder $\mathrm{MnO}$ ) vor Feuchtigkeit und Sauerstoff geschützt und so die Lagerfähigkeit verlängert werden. Das Patent wurde bislang nicht eingesetzt, soll in verschiedenen Ländern auslaufen und nicht mehr ernevert werden. ${ }^{22} \mathrm{TiO}_{2}$ in Nanoform ist für Lebensmittel nicht zugelassen. Bislang gibt es keine gesicherten Hinweise darauf, dass es derzeit in Lebensmitteln eingesetzł wird. ${ }^{18}$

(6) Zerkleinerung von Heilkräutern auf Nanogröße (Nanopulver, Nanoemulsionen; in Anwendung, z. B. in Nahrungsergänzungsmitteln).

(7) Wasseraufbereitung mittels Nano-Membranen (Desinfektion, Schadstoffentfernung; in Entwicklung).

(8) Katalytische Verbesserung von Frittieröl durch einen nanokeramischen Einsatz für Gewerbe-Fritteusen, welcher die Polymerisationen des Öls und die damit verbunde-

Tabelle 1: Nanopartikel und nanostrukturierte Materialien in der Lebensmittelindustrie und Anwendungsbeispiele

\begin{tabular}{|c|c|c|}
\hline Kategorie & $\begin{array}{l}\text { Nanopartikel, } \\
\text { nanostrukturierte Materialien }\end{array}$ & Anwendungsbeispiele \\
\hline \multirow[t]{8}{*}{$\begin{array}{l}\text { Lebensmittel- } \\
\text { herstellung }\end{array}$} & $\begin{array}{l}\text { Nanokapseln (Mizellen, Liposome) } \\
\text { Nanoemulsionen } \\
\text { Nanoröhren und Nanokugeln } \\
\text { aus Milchprotein }\end{array}$ & $\begin{array}{l}\text { Verkapselung, Verbesserung der Löslichkeit, } \\
\text { Schutz und kontrollierte Abgabe von } \\
\text { Inhaltsstoffen; Anwendung z. B. für } \\
\text { "Nutraceuticals" bzw. "Functional Food" }\end{array}$ \\
\hline & Membrane & Wasseraufbereitung, Filter \\
\hline & nanokeramische Beschichtungen & Reinigung und Frischhaltung von Frittieröl \\
\hline & Nanopulver & Heilkräuterzusätze \\
\hline & Nano-Lycopin & $\begin{array}{l}\text { Antioxidans für Nahrungsergänzungs- } \\
\text { und Lebensmittel }\end{array}$ \\
\hline & Nano-Beta-Carotin & Farbstoff für Getränke \\
\hline & Nano-Siliziumdioxid & $\begin{array}{l}\text { Lebensmittelzusatzstoff und } \\
\text { Verarbeitungshilfsstoff }\end{array}$ \\
\hline & $\mathrm{TiO}_{2}, \mathrm{SiO}, \mathrm{CaO}, \mathrm{ZnO}, \mathrm{MnO}$ & Überzug von Süßwaren \\
\hline Verpackung & $\begin{array}{l}\text { Nanokomposite, Nanopartikel } \\
\text { (Silber, Titandioxid, Siliziumdioxid, } \\
\text { Nano-Ton) }\end{array}$ & $\begin{array}{l}\text { Folien, Verpackungscontainer, } \\
\text { PET-Flaschen }\end{array}$ \\
\hline \multirow[t]{2}{*}{$\begin{array}{l}\text { Sicherheit und } \\
\text { Sensortechnik }\end{array}$} & $\begin{array}{l}\text { Nano-Cantilever (Messnadeln) } \\
\text { Nanodrähte }\end{array}$ & $\begin{array}{l}\text { Sensoren zum Aufspüren von } \\
\text { Krankheitserregern, Chemikalien, Giften }\end{array}$ \\
\hline & Nano-Silber & $\begin{array}{l}\text { Antibakterielle Beschichtungen in } \\
\text { Haushaltsgeräten }\end{array}$ \\
\hline
\end{tabular}

nen schlechten Gerüche verhindert (Produkt OilFresh, USA).

(9) Filterpapier mit Nano-Silizium zur Entfernung von Coffein aus dem Kaffee (in Entwicklung; Universität von Nebraska, Lincoln, USA). ${ }^{23}$ Ähnliche Filterpapiere könnten auch zur Entfernung von Cholesterin oder Nikotin eingesetzt werden.

(10) Interaktive Lebensmittel und Getränke mit Nanokapseln, die bei bestimmten Mikrowellen-Frequenzen aufbrechen und die gewünschten Aromen und Farben freisetzen ("on-demand-delivery"). Dadurch wird eventuell in Zukunft die in-situ-Modifikation eines Produktes hinsichtlich Geschmacks und Farbe ermöglicht. Ein bekanntes Beispiel dafür ist die so genannte "Magic Pizza", die aber bislang noch ein reines Phantasieprodukt ist.

\section{Verpackung}

Nanokomposite 24 oder Nanopartikel (z. B. Silber, $\mathrm{TiO}_{2}, \mathrm{SiO}_{2}$, Nano-Ton) in Verpackungsmaterialien können einen besseren Schutz der Lebensmittel gewährleisten, etwa indem sie die Durchlässigkeit von Folien verringern, desodorierend wirken, UV-Licht abblocken, die Hitzebeständigkeit und die Beständigkeit gegenüber mechanischer Belastung erhöhen, gegen Bakterien oder Pilze wirken.
$\mathrm{TiO}_{2}$ in Nanoform ist transparent, behält aber seine UV-Beständigkeit und wird von mehreren Firmen als Füllpartikel in Folien und Plastikcontainern vertrieben. $\mathrm{Nano}_{-} \mathrm{TiO}_{2}$ in Verpackungsmaterialien wird als lebensmittelsicher eingestuft, obwohl sich die meisten Sicherheitsdaten auf die größere Form beziehen. Es ist nicht bekannt, ob es Untersuchungen dazu gibt, dass Verpackungsmaterialien mit $\mathrm{Nano}^{-\mathrm{TiO}_{2}}$ ebenso inert (wenig reaktiv) sind, wie jene mit der größeren „Bulk"Form, d. h. es ist nicht auszuschließen, dass Nano-TiO 2 -Partikel von der Verpackung auf die Lebensmitteln übergehen.

Nylon-Nanokomposite werden für Lebensmittelverpackungen verwendet (z. B. PETFlaschen für Bier und andere alkoholische Getränke), da sie eine Barriere für Saverstoff und $\mathrm{CO}_{2}$ darstellen und damit die Frische des Produktes erhalten bleibt bzw. üble Gerüche abgehalten werden.

\section{Sicherheit und Sensortechnik}

(1) Protein-umhüllte Nano-Cantilever (Messnadeln), die bei einer spezifischen Frequenz vibrieren, stellen eine neue Klasse von ultrakleinen Sensoren dar, die derzeit entwickelt werden. Sie sollen ein rasches Aufspüren von Viren, Bakterien und anderen Krankheitserregern ermöglichen. Wenn Verunreinigungen (etwa Bakterien) an der spezifizierten Ober- 
fläche haften bleiben, führt das zu einer Massenänderung des Cantilevers und damit zu einer nachweisbaren Verschiebung der Resonanzfrequenz. ${ }^{25}$

(2) Nanodrähte, versehen mit synthetischer "baumförmiger" DNA (Dendrimere), könnten in Zukunft als eine Art Nano-Strichcode fungieren, um Krankheitserreger in Lebensmitteln aufzuspüren. ${ }^{26}$

(3) Ein tragbarer Miniatur-Mikrobiodetektor wurde unter Verwendung verschiedener Nanodrähte sowie spezifischer, fluoreszierender Antikörper in den USA entwickelt, um gleichzeitig Gifte, Krankeitserreger und Chemikalien aufzuspüren. Das spezielle Einsatzgebiet dieses Gerätes sind Biowaffen (z. B. Anthrax). Es kann aber in Zukunft auch im Bereich der Lebensmittelsicherheit von Interesse sein. 27

(4) Beschichtungen mit Silber-Nanopartikeln werden bereits für Kühlschränke verwendet, um die Ausbreitung von Bakterien und Mikroben zu verhindern.

Tabelle 1 gibt einen Überblick über die Anwendungsbereiche und verwendeten Nanomaterialien.

\section{Nanotech im Handel}

\section{Nanolebensmittel}

Das Woodrow Wilson Center 28 listet 67 Produkte in der Kategorie "Food \& Beverages", von denen die Hersteller explizit behaupten, dass es sich um nanotechnologisch hergestellte Lebensmittel oder Lebensmittel mit nanoskaligen Komponenten handelt (Stand vom 29.2.08). Darunter finden sich: antibakterielles Besteck, Anti-Haft-Pfannen, Desinfektionsspray, Reinigungsmittel, Backformen, gasdichte PET-Flaschen, Aufbewahrungsbehälter, Kühlschränke, Babyflasche, Folien sowie vierzig Einträge unter "Nahrungsergänzungsmittel". Nur drei Artikel sind Nahrungsmittel:

- Canola Active Oil, Israel: (Mizellen mit Phytosterol behindern die Aufnahme von Cholesterin im Darm).

- Nanoceuticals Slim Shake Chocolate, USA: Schlankheitsmittel mit "Nanoclusters" (Delivery-System) für den Schokoladegeschmack.

\section{- Nanotea, China: Tee mit Nano-Selen.}

Die Produktliste www.nanoproducts. de listet zehn Produkte aus dem Lebensmittelbereich (Stand 9.4.08). Darunter zwei Industriepro- dukte (Nanokapseln), sieben Nahrungsergänzungsmittel (Mineralien, Zeolith, kolloidales Silber, Co-Enzym Q10) sowie Nanotee mit Selen.

Weitere Beispiele sind:

- Kaugummi mit verkapseltem Kakaopulver für den Schokoladegeschmack, O'lala Foods (USA). ${ }^{29}$

- Tip-Top Up Bread (Brot mit Nanokapseln für Fischöl), Nu-Mega and Clover Corp. (Australien): Die Kapseln schützen das Fischöl vor Oxidation und verhindern den unangenehmen Fischgeschmack. Die Konsumentlnnen profitieren von den gesundheitlichen Vorteilen der im Fischöl enthaltenen Omega-3-Fettsäuren. 30

\section{Nahrungsergänzungsmittel}

Im Bereich Nahrungsergänzungsmittel finden sich zumeist nanoskalige Mineralstoffe wie Silicium, Magnesium, Calzium, Zeolith aber auch Nanosilber und Nanogold.

Das Woodrow Wilson Center listet 40 Nahrungsergänzungsmittel, d. i. die Mehrheit der 67 Produkte, die unter der Kategorie „Food \& Beverages" angeführt sind. ${ }^{31}$

Einer der Anbieter von Nahrungsergänzungsmitteln mit Nanomineralien ist die Firma "Neosino", deren Produkte auch in Österreich erhältlich sind. Silizium, Magnesium und Calcium in Nanoform sollen eine bessere Bioverfügbarkeit aufweisen und insbesondere der Stärkung des Immunsystems dienen. ${ }^{32}$ Auch Nahrungsergänzungsmittel für Bodybuilder sind im Handel erhältlich, z. B. NaNO Vapor der Firma Margaritella ${ }^{33}$, ebenso wie eine Reihe von Produkten, die von Online-Händlern direkt über das Internet vertrieben werden. Dazu gehören z. B.

\section{Fazit}

Das zunehmende Interesse am Thema Nanotechnologie im Lebensmittelbereich ist angesichts einer Vielzahl von Nanopartikeln und nanostrukturierten Materialien, die bereits zum Einsatz kommen und weltweit gehandelt werden, durchaus berechtigt. Aufgrund der geringen Bereitschaft der Industrie ihre Entwicklungen und Anwendungen offen zu kommunizieren, bleiben viele Fragen ungeklärt. Mehr Transparenz und ein Dialog mit der Öffentlichkeit könnten dazu beitragen, Unklarheiten zu beseitigen und eine fundierte Auseinandersetzung sowohl mit den Vorteilen als auch mit potenziellen Risiken zu fördern. die Produkte aus der NanoPRIME Linie, die Nano-Zeolith enthalten und bei einer Vielzahl von Erkrankungen helfen sollen. ${ }^{34} \mathrm{Kol}-$ loidales Silber- und Goldwasser kann ebenfalls über das Internet bezogen werden und verspricht neben Glück und Harmonie auch die Heilung verschiedenster Krankheiten. ${ }^{35}$

\section{Anmerkungen und Literaturhinweise}

1 Institute of Food Science \& Technology (IFST) (2006): Nanotechnology Information Statement.

2 US5741505, 25.7.1996.

3 www.wikipatents.com/ca/2210851.html.

4 Verkapselungssysteme bieten Schutz und dienen dem Wirkstofftransport und der Wirkstoffabgabe durch sogenannte Nanokapseln (Mizellen, Liposome).

5 Ubbink J. und Krüger J. (2006): Physical approaches for the delivery of active ingredients in foods. Trends in Food Science \& Technology $17,244-254$.

6 Bundesinstitut für Risikobewertung (2007): The majority of consumers view the development of nanotechnology favourably. Pressemitteilung 19.12.2007, www.bfr.bund.de/cd/10563.

7 www.milchindustrie.de.

8 Nach Chen H., Weiss J., Fereodoon S. (2006): Nanotechnology in Nutraceuticals and Functional Food. Emerging technology has shown great potential for delivering bioactive compounds in functional foods to improve human health. foodtechnology 03.06, pp. 30-36.

9 Functional Food: funktionelle Lebensmittel, welche mit zusätzlichen Inhaltsstoffen („Nutraceuticals") angereichert werden, die einen positiven Effekt auf die Gesundheit haben sollen.

10 Nutraceuticals: Kombination der englischen Wörter "Nutrition" (Ernährung) und "Pharmaceuticals" (Pharmazeutika). Nutraceuticals sind biologisch aktive Inhaltsstoffe (z. B. sekundäre Pflanzenstoffe, Antioxidantien), die im Bereich des "Functional Foods" eingesetzt werden und einen medizinischen bzw. gesundheitlichen Nutzen haben sollen (z. B. Senkung des Cholesterinspiegels).

11 Nach Chau Ch., Wu S., Yen G. (2007): The development of regulations for food nanotechnology. Trends in Food Science \& Technology 18 (2007), pp. 269-280. Aufzählung im Text verändert und ergänzt.

12 www.media.basf.com/de/presse/ mitteilungen $/ \mathrm{pm} \cdot \mathrm{htm}$ ? pmid $=2961 \mathrm{\& n}=$ 10\&id = V00-XxSb3C5RTbcp*e-, 8.4.08.

13 BASF Presseinformation, „Beta-Carotin für Getränke: Erst die Formulierung sorgt für Farbe und Stabilität", Dr. Lutz End,

corporate.basf.com/de/innovationen/felder/ ernährung.

14 www.zusatzstoffe-online.de. 
15 Anhang zur Aktualisierung des Anhanges $\mathrm{VI}$ der EU-VO 2092/91 - Änderung vom 25.5. 2006.

16 www.aerosil.com/aerosil/de.

17 www.salinen.at.

18 Grobe A., Renn O. \& Jäger A. (2008): IRGC workshop briefing paper: Appropriate risk governance strategies for nanotechnology applications in food and cosmetics. International Risk Governance Council (IRGC).

19 Dazu wörtlich eine Information von Evonik (www.degussa-nano.com/nano/de/nachhaltigkeit/sicherereprodukte): "Amorphe Kieselsäure wird seit Jahrzehnten als Verarbeitungshilfsmittel in Trockenpulver in die Lebensmittelindustrie verkauft. Typische Einsatzgebiete sind der Zusatz zu Gewürzpulver, Tomatenpulver oder Eipulver. Der Mengenanteil im Trockenpulver liegt im unteren einstelligen Prozentbereich. Kieselsäure ist für diese Anwendung geprüft und als Lebensmitteladditiv E 551 seit den 60er Jahren zugelassen. Struktur und Teilchenfeinheit der in Lebensmitteln verwendeten Kieselsäure wurden bis zum heutigen Tag nicht geändert. Umfangreiche toxikologische Studien geben keine Hinweise auf nachteilige gesundheitliche Effekte. Auch bei der langiährigen Verwendung von pyrogenen Kieselsäuren als Zusatz in vielen Arzneimitteln sind keine Auffälligkeiten bekannt."

20 US5741505, 25.7.1996.

21 www.wikipatents.com/ca/2210851.html.

22 BUND (Freunde der Erde) (2007): Aus dem Labor auf den Teller.
23 Goho A. (2004): Hungry for nano: the fruits of nanotechnology could transform the food industry. Science News, 25.9.2004.

24 Nanokomposite: Verbundwerkstoffe, bei denen Nanopartikel in eine Matrix eingebunden sind.

25 ElAmin Ahmed (2006): Nanocantilevers studied for quick pathogen detection. www.nutraingredients.com/news/ ng.asp? $\mathrm{n}=70159$-nanotechnologynanoscale-pathogens.

26 Color coded pathogens offer safer food formulation (2005), www.foodnavigator.com/ news/news-ng.asp? $\mathrm{n}=60665$-colour-codedpathogens.

27 Codierte Nano-Drähte entlarven Biowaffen (2007). www.lifegen.de/newsip/ shownews.php4? getnews $=2006-09-08-51$ $11 \& \mathrm{pc}=\mathrm{s} 01$

28 www.nanotechproject.org.

29 www.olalafoods.com, www.foodprocessing.com/articles/2006/ 227.html.

30 www.nu-mega.com; www.foodprocessing.com/articles/2006/ 227.html.

31 www.nanotechproject.org, Zugriff 29.2.08.

32 www.neosino.at.

33 www.margaritella.com.

34 www.nanoprime.at.

35 www.gela.at.
IMPRESSUM:

Medieninhaber: Österreichische Akademie der Wissenschaften; Juristische Person öffentlichen Rechts (BGBI 569/1921 idF BGBI I 130/2003); Dr. Ignaz Seipel-Platz 2, A-1010 Wien

Herausgeber: Institut für Technikfolgen-Abschätzung (ITA); Strohgasse 45/5, A-1030 Wien; www.oeaw.ac.at/ito

Erscheinungsweise: Die NanoTrust-Dossiers erscheinen unregelmäßig und dienen der Veröffentlichung der Forschungsergebnisse des Instituts für Technikfolgen-Abschätzung im Rahmen des Projekts NanoTrust. Die Berichte werden ausschließlich über das Internetportal „epub.oeaw” der Öffentlichkeit zur Verfügung gestellt: epub.oeaw.ac.at/ita/nanotrust-dossiers/

NanoTrust-Dossier Nr. 004, Mai 2008: epub.oeaw.ac.at/ita/nanotrust-dossiers/dossier004.pdf

ISSN: 1998-7293

Dieses Dossier steht unter der Creative Commons

SOMIEAIGHISHESERVED (Namensnennung-NichtKommerziell-KeineBearbeitung 2.0 Österreich)

Lizenz: creativecommons.org/licenses/by-nc-nd/2.0/at/deed.de 


\section{nano dossiers}

\section{Ergänzung zu Dossier Nr. 004, Stand: Dezember 2010}

Die im Text genannten Produkte „Canola Active Oil“ (Israel), „Tip-Top Up Bread“ (Australien) und „NanoPRIME“ (Österreich) sind nicht mehr am Markt erhältlich. Das Produkt LycoVit (nanopartikuläres synthetisches Lycopin von BASF) wurde 2009 als Lebensmittelzusatzstoff unter der Novel-Food-Verordnung (258/97) in der EU zugelassen. Das Schlankheitsprodukt "Nanoceuticals Slim Shake Chocolate“ wurde in "Slim Shake Chocolate" umbenannt. Der Hersteller gibt keine Informationen mehr zu möglichen nanopartikulären Inhaltsstoffen dieses Produkts auf seiner Webseite (www.rbclife.com). Die Firma Margaritella führt laut ihrer Webseite das Nahrungsergänzungsmittel "NaNO Vapor" nicht mehr. Stattdessen gibt es nunmehr das Produkt naNOX9, das laut Herstellerangaben auf der „Nanomolecular Rapid Explosion Technology ${ }^{\top м} "$ beruht (www.margaritella.com). Nano-Membrane werden mittlerweile bei der Wasserreinigung und -aufbereitung eingesetzt.

Die EU-Novel-Food-Verordnung (258/97) befindet sich derzeit in Revision und soll neben der Definition von Nanomaterialien auch ein spezielles Zulassungsverfahren für Lebensmittel mit Nanomaterialien sowie eine Kennzeichnungspflicht vorsehen (siehe dazu auch NanoTrust Dossier Nr. 017, April 2010).

\section{Addendum for Dossier No. 004, Version: December 2010}

The following products, mentioned in this paper, are no longer available on the market: „Canola Active Oil“ (Israel), „Tip-Top Up Bread“ (Australia) und „NanoPRIME“ (Austria). In 2009 LycoVit (nanoparticulate synthetic lycopene, BASF) was approved under the Novel Food Regulation (258/97) of the European Union as a novel food ingredient. The slimming product "Nanoceuticals Slim Shake Chocolate" was renamed in "Slim Shake Chocolate". The company offers no longer information on its website regarding possible nanoparticulate ingredients of this product (www.rbclife.com). According to its website, the company Margaritella no longer offers "NaNo Vapor", a food supplement for bodybuilders. Instead there is now "naNoX9" available, which bases according to information of the company, on the "Nanomolecular Rapid Explosion Technology ${ }^{{ }^{\mathrm{N}} \text { “ }}$ (www.margaritella.com). Nano-membranes are already in use for water-purification.

The EU Novel-Food-Regulation (258/97) is currently under revision and shall include a definition of nanomaterials, a specific authorisation procedure as well as labelling of products containing nanomaterials (see NanoTrust Dossier No. 017en, November 2010). 